\title{
Gestión comunicativa de crisis de las oficinas nacionales de turismo de España e Italia ante la Covid-19
}

\author{
Crisis communication management by the national \\ tourist organizations of Spain and Italy in \\ the face of Covid-19
}

\author{
Assumpció Huertas; Andrea Oliveira; Michele Girotto
}

Cómo citar este artículo:

Huertas, Assumpció; Oliveira, Andrea; Girotto, Michele (2020). "Gestión comunicativa de crisis de las oficinas nacionales de turismo de España e Italia ante la Covid-19”. Profesional de la información, v. 29, n. 4, e290410. https://doi.org/10.3145/epi.2020.jul.10

Artículo recibido el 06-05-2020 Aceptación definitiva: 17-05-2020

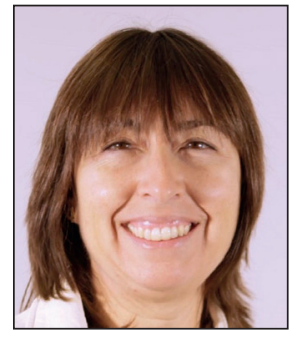

Assumpció Huertas https://orcid.org/0000-0001-6684-4220

Universitat Rovira i Virgili

Estudios de Comunicación

Av. Catalunya, 35.

43002 Tarragona, España

sunsi.huertas@urv.cat

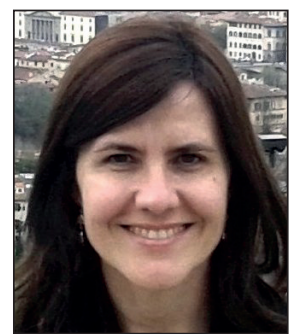

\section{Michele Girotto}

https://orcid.org/0000-0001-6046-7233

Universitat de Barcelona

Facultat d'Economia i Empresa

Dept. d'Empresa

Av. Diagonal, 690.

08034 Barcelona, España

michele.girotto@ub.edu

\section{Resumen}

La crisis sanitaria de la Covid-19 está afectando a diversos sectores económicos, especialmente al turístico. Esta investigación persigue conocer cómo las oficinas nacionales de turismo (ONTs) de España e Italia gestionaron su comunicación de crisis ante la Covid-19. El estudio se llevó a cabo mediante el análisis de contenido de los mensajes publicados en las cuentas de Twitter de las ONTs desde el inicio o la detección del primer paciente hasta un mes después de la declaración oficial de pandemia por la Organización Mundial de la Salud. Los resultados revelan dos maneras distintas de administrar la comunicación de crisis ante la Covid-19 tanto en el tratamiento de los temas como en los tiempos de la publicación, que a la vez generan distintas reacciones y engagement entre los usuarios, siendo la Agenzia Nazionale Italiana del Turismo más rápida y activa en el primer momento de la crisis en comparación con la Oficina de Turismo de España. Este estudio es una contribución para las ONTs de los países que sufren crisis sanitarias en la creación de sus estrategias comunicativas en los medios sociales, tanto en los diferentes períodos de la crisis como respecto a las acciones comunicativas para recuperar la imagen turística de los destinos.

\section{Palabras clave}

Covid-19; Coronavirus; Pandemias; Relaciones públicas; Comunicación de crisis; Estrategias de comunicación; Turismo; Oficinas nacionales de turismo; ONT; Medios sociales; Redes sociales; Twitter.

\section{Abstract}

This study analyzes how the national tourism organizations (NTOs) of Spain and Italy managed their crisis communication to deal with Covid-19. The study examines the messages published by the Twitter accounts of the NTOs from the 
beginning or the detection of the first patient until one month after the official declaration of the pandemic by the World Health Organization. The results reveal two different ways of managing crisis communications in the face of Covid-19, both in the treatment of the topics and in the timing of publication, at the same time generating different reactions and engagement among users. The Agenzia Nazionale Italiana del Turismo was faster and more active in the first moments of the health crisis compared with the Oficina de Turismo de España. This study can contribute to the development of communication strategies on social media by NTOs during different periods of such crises as well as communication actions to enhance the touristic image of their destinations.

\section{Keywords}

Covid-19; Coronavirus; Pandemics; Public relations; Crisis communication; Communication strategies; Tourism; National tourism organizations; Tourism offices; NTOs; Social media; Social networks; Twitter.

\section{Introducción}

La Covid-19, la enfermedad vírica que apareció en Wuhan (China) en 2019, se ha convertido en una pandemia mundial que ha afectado a más de 190 países y ha comportado el cierre de fronteras, la anulación de vuelos y el confinamiento de las personas en sus casas (Yang; Zhang; Cheng, 2020). Estas medidas gubernamentales, que se han tomado en la mayoría de países para evitar o frenar la propagación de esta enfermedad tan contagiosa, suponen una drástica restricción de la movilidad humana, con consecuencias directas en el sector turístico.

En las últimas dos décadas ya surgieron pandemias producidas por enfermedades víricas y contagiosas como: el SARS de 2002-2003 que surgió en China, la gripe A de 2009 (H1N1) en Méjico, el coronavirus (MERS.Cov) que surgió en 2012 en Arabia Saudí, el Ébola (2013-2016) en Guinea o el Zika (2015) en Brasil. Su expansión y sus consecuencias fueron diversas, afectando en distinto grado a la movilidad humana, la situación económica y al turismo. De hecho, dado que se ha demostrado que el transporte aéreo (Browne et al., 2016) y los viajes (Gardner; Chughtai; Maclntyre, 2016) son destacados factores de propagación de estas enfermedades, las medidas que se adoptan van en la línea de prohibir todo ello. Pero ninguna pandemia anterior había comportado medidas tan restrictivas para la movilidad humana en tantos lugares.

Los dos países europeos donde más ha afectado la Covid-19 en el inicio de la pandemia son Italia y España, los cuales a finales de abril de 2020 sumaban entre los dos el 44\% de los muertos mundiales por esta enfermedad (Saura, 2020). Las causas de esta superior afectación en estos dos países se explican por

- la elevada calidad de vida y envejecimiento de la población que poseen, ya que esta enfermedad afecta precisamente a los mayores;

- las costumbres y la sociabilidad mediterránea, que suponen un superior contacto entre las familias;

- la falta de tests y la reacción tardía de las medidas gubernamentales.

Por otro lado, España e Italia son dos países con una elevada demanda turística y un gran peso del turismo en los PIBs nacionales.

Ante esta situación, las extremas medidas adoptadas por los gobiernos de confinamiento de los ciudadanos y de la prohibición de la movilidad humana han supuesto un grave impacto y una crisis sin precedente para el sector turístico, que escapa a las decisiones de las oficinas nacionales de turismo (ONTs). Las medidas adoptadas han restringido totalmente las actividades lúdicas, de ocio y turísticas y han comportado también incertidumbre respecto a cuándo y cómo se podrá viajar. Además, la cobertura sensacionalista y en ocasiones racista y discriminatoria de los medios de comunicación de masas también genera unos efectos para la imagen de los países que afecta a su demanda turística (Wen et al., 2020). Dada esta situación crítica para el sector turístico, las ONTs deben gestionar una correcta comunicación de crisis, que les ayude a generar confianza y seguridad (Oliveira; Huertas, 2019), a reforzar su imagen y reputación (Xifra, 2020) y a crear el deseo de visitar el país cuando sea permitido.

Los medios tradicionales de comunicación de masas, que han recuperado protagonismo y audiencia en esta crisis de la Covid-19 (Casero-Ripollés, 2020), suelen ofrecer una información demasiado alarmista o sensacionalista sobre estas pandemias o crisis sanitarias (Marco; López, 2010). Pero los medios sociales son fundamentales para la gestión de la comunicación de crisis en momentos de incertidumbre generados por las pandemias (Veil; Buehner; Palenchar, 2011; Chew et al., 2013). Gui et al. (2017) incluso demostraron que los medios sociales y en especial Twitter y Facebook fueron clave para tomar las decisiones turísticas de los usuarios ante la pandemia del Zika en 2015.

Por todo ello, el objetivo de este estudio es conocer cómo las ONTs de España e Italia estuvieron gestionando la comunicación de crisis a través de sus cuentas de Twitter para informar a las personas interesadas y turistas potenciales, crear o recuperar la imagen turística de los países, comunicar seguridad y generar atracción turística.

La crisis sanitaria de la Covid-19 está afectando especialmente al sector turístico 


\section{Marco teórico}

\subsection{Pandemias y crisis sanitarias y su impacto en el turismo}

Todas las pandemias que han tenido lugar en el siglo XXI han provocado crisis económicas y han tenido su impacto tanto en las decisiones turísticas como en el comportamiento de los turistas (Lee et al., 2012), generando crisis de diversa gravedad en el sector. Cabe destacar que todas las crisis tienen el potencial de causar daños emocionales, físicos, financieros y medioambientales a los públicos afectados (Freberg; Palenchar; Veil, 2013). Debido a que el turismo supone una elevada fuente de ingresos y un generador de desarrollo económico para los países (Lee; Chang, 2008), las crisis turísticas también tienen un gran efecto en sus economías (Shi; Li, 2017).

Los medios de comunicación de masas han dado una gran cobertura a las pandemias y su tratamiento ha generado percepción social de riesgo, un aumento del miedo y desconfianza entre la población (Marco; López, 2010). Concretamente en la prensa española, la metáfora bélica utilizada mayoritariamente por los periódicos en la información sobre la crisis sanitaria de la gripe A (H1N1) del 2009 generó mucho miedo, alarma y ansiedad entre los ciudadanos (Marco; López, 2011), al igual que ocurrió en otros países (Maciel-Lima et al., 2015). El tratamiento actual de la Covid-19, que ha comportado un mayor seguimiento de las noticias por todos los públicos a través de los medios de comunicación tradicionales (Casero-Ripollés, 2020), ha tenido el mismo efecto.

Como otros estudios han demostrado previamente, la demanda turística es muy sensible a cualquier tipo de riesgo (Fuchs et al., 1986). De hecho, ante el menor riesgo percibido en los destinos, los potenciales turistas cambian de destino o modifican sus decisiones turísticas (Araña et al., 2009). De igual modo, los turistas que perciben el riesgo de contagiarse una enfermedad durante un viaje decidirán posponerlo o cancelarlo para evitar el riesgo de contagio (Reisinger; Mavondo, 2005). El SARS de 2002-2003, que apareció en China y se extendió por todo el mundo rápidamente (Varia et al., 2003), generó advertencias sanitarias de no viajar a determinados países asiáticos, y ello comportó la pérdida de miles de puestos de trabajo en el sector turístico asiático (McKercher; Chon, 2004). Diversos estudios analizaron su impacto en el sector turístico (Henderson; Ng, 2004; Kim; Chun; Lee, 2005; Wang, 2009).

La pandemia de la gripe A de 2009 (H1N1) impactó de forma importante en el turismo internacional de 2010. En todos los continentes excepto África y Sudamérica la demanda turística disminuyó (Lee et al., 2012). Lee et al. (2012) demostraron que en los 5 países en que los gobiernos adoptaron medidas más restrictivas para la propagación del virus como la cuarentena de los pacientes, el cierre de escuelas, la cancelación de eventos públicos o el control de las fronteras internacionales, es donde el turismo más disminuyó. Hung et al. (2018) analizaron el rol de la industria turística en respuesta a las crisis sanitarias del SARS (2003) y H1N1 (2009). El coronavirus (MERS.Cov) que surgió en el 2012 en Arabia Saudí también tuvo su impacto en el sector turístico. Dado que la enfermedad impactó especialmente en Corea del Sur, esta sufrió un drástico descenso de la demanda turística (Joo et al., 2019), especialmente de China, que es su principal país emisor de turistas potenciales (Shi; Li, 2017).

Como se observa, todos los estudios citados han analizado el impacto de estas pandemias en la industria turística, pero estudios sobre la gestión de la comunicación de crisis realizada por las ONTs de los países son prácticamente inexistentes. Jha et al. (2018) analizaron la comunicación de crisis de las administraciones públicas gubernamentales ante las crisis sanitarias y destacaron las mejores prácticas adoptadas en estos casos, pero se centraron en la comunicación de las pandemias y la salud, no en el sector turístico. En cambio, Ritchie et al. (2004) sí estudiaron la gestión de la comunicación de crisis de la autoridad turística británica después de la crisis sanitaria del 2001.

\subsection{El rol de los medios sociales y de Twitter en la comunicación de crisis de las pandemias en el ámbito turístico}

Los medios sociales juegan un importante rol en la comunicación de crisis (Veil; Buehner; Palenchar, 2011), tanto en su uso por parte de las organizaciones que son creadoras de contenido y les interesa gestionar una excelente comunicación de crisis, como por parte de los públicos, seguidores o inactivos, que reciben y también difunden información (Liu; Austin; Jin, 2011). Por tanto, las organizaciones ya no controlan de igual modo la comunicación de crisis y los públicos ahora han tomado protagonismo con el electronic word-of-mouth (eWOM) (Colley; Collier, 2009), participando activamente en la comunicación durante las crisis (Cheng, 2020; Romenti; Murtarelli; Valentini, 2014). Así pues, la social-mediated crisis communication (Cheng, 2020) o la comunicación de crisis a través de los medios sociales, es una comunicación más completa y útil para los usuarios (Gui et al., 2017) porque se crea no solo a través de la participación de todos los públicos sino también de la visión de las organizaciones.

Twitter destaca entre todos los medios sociales como un medio particularmente idóneo para la comunicación de las crisis (Brummette; Sisco, 2015) por sus características y singularidades. En primer lugar, su gran capacidad interactiva permite a las organizaciones establecer relaciones de calidad con los públicos (Huertas; Setó-Pàmies; Míguez-González, 2015; Saffer; Sommerfeldt; Taylor, 2013); facilita información en tiempo real (Brummette; Sisco, 2015) y ofrece soporte emocional y comunitario (Saffer; Sommerfeldt; Taylor, 2013). Además, la comunicación de crisis vía Twitter genera reacciones menos negativas en los públicos que la información de crisis recibida por otros canales como los blogs o los periódicos (Schultz; Utz; Göritz, 2011). 
Los medios sociales han transformado la forma en que las organizaciones sanitarias o de cualquier tipo comunicaban a los públicos las enfermedades infecciosas y el riesgo que comportan (Vijaykumar; Jin; Nowak, 2015). Los medios sociales jugaron un importante rol en la pandemia H1N1, que fue la primera en la era de los medios sociales (Vijaykumar; Jin; Nowak, 2015). Por ello muchos estudios han analizado el rol de los medios sociales en su comunicación (Freberg; Palenchar; Veil, 2013; Liu; Kim, 2011; Signorini; Segre; Polgreen, 2011; Vijaykumar; Jin; Nowak, 2015). De hecho, Twitter registró más de 2 millones de tweets durante esta pandemia en 2009 (Chew; Eysenbach, 2010).

Otros estudios, como el de Gui et al. (2017), analizaron cómo los turistas potenciales utilizaron los medios sociales durante la pandemia del Zika para tomar sus decisiones de viaje. Debido a la desinformación reinante durante la crisis del Zika, y debido a que las autoridades no informaron suficientemente sobre turismo, los turistas buscaron la información de residentes locales y de turistas que habían estado previamente en los destinos a través de los medios sociales para posteriormente tomar sus decisiones de viaje.

Las organizaciones deben responder a las crisis con una gestión de la comunicación de crisis exitosa (Boin; Lagadec, 2000), restaurando el orden normal y satisfaciendo las necesidades de información y las expectativas de los públicos a través de un uso efectivo de los medios sociales. De hecho, los medios sociales permiten a las organizaciones en momentos de crisis monitorizar las opiniones y los sentimientos de los públicos, destruir rumores, establecer relaciones favorables y mantener su credibilidad (Veil; Buehner; Palenchar, 2011). Además, generan transparencia para las acciones de las organizaciones (Cheng, 2020; Jin; Liu, 2010).

A pesar de que la crisis de la Covid-19 genera baja responsabilidad para las organizaciones (Xifra, 2020), estas deben dar respuesta a la situación para mantener su imagen. Muchos son los estudios científicos que están surgiendo sobre la Covid-19 (Torres-Salinas, 2020), la mayoría desde el ámbito sanitario y social. Thelwall y Levitt (2020) analizaron la comunicación de la crisis sanitaria de la Covid-19 a través de Twitter, pero desde el ámbito sanitario y no turístico. Dado que esta crisis del coronavirus afecta tanto al sector del turismo, y en especial a países tan turísticos como España e Italia, sus ONTs deben realizar una correcta comunicación de crisis a través de los medios sociales y Twitter.

\section{Metodología}

Así pues, este estudio analiza los contenidos publicados por las ONTs de España e Italia en sus cuentas oficiales de Twitter, con el objetivo de identificar sus prácticas comunicativas online en la gestión de la crisis de la Covid-19 y valorar si su comunicación persigue informar a los stakeholders y turistas potenciales de la crisis, crear o recuperar la imagen turística de los países, comunicar seguridad y generar atracción turística. Como objetivos secundarios el estudio se plantea conocer con qué rapidez se comunicaron y publicaron las ONTs en Twitter, si publicaron tweets propios o retweetearon los existentes de otras entidades y qué éxito obtuvieron sus tweets entre los usuarios a partir de las reacciones que generaron.

\subsection{Casos de estudio}

Se seleccionaron España e Italia porque son los dos países de Europa donde la pandemia más estuvo impactando en la fase inicial, a finales de abril de 2020 (Saura, 2020). Pero además, también se seleccionaron por la importancia del turismo en estos países. Concretamente son el segundo país del mundo, España, y el quinto, Italia, en número de llegadas de turistas internacionales (Europa Press, 2019).

España logró un récord de turistas extranjeros en 2019, con 83,7 millones de viajeros internacionales y un gasto de 92.337 millones de euros, que supone un incremento del 3,2\%. En España el turismo aportaba el 12,3\% del PIB y el 12,7\% del empleo (Molina, 2020). Y en Italia en 2017 por primera vez el número de turistas procedentes del extranjero superó al de los locales, y los datos del sector en 2019 mostraron un aumento del 4\% en las llegadas de turistas internacionales (Ansa, 2020).
Esta investigación analiza la comunicación de las ONTs de España e Italia ante la Covid-19 a través de Twitter 


\subsection{Preguntas de investigación, recopilación y análisis de los datos}

Para alcanzar los objetivos del estudio, se establecieron 5 preguntas de investigación:

PI1: ¿Cuáles son los principales contenidos de los tweets de las ONTs de España e Italia durante el primer periodo de crisis de la Covid-19 en Twitter?

PI2: ¿Con qué timing las dos ONTs comunicaron la crisis de la Covid-19 a través de sus cuentas en Twitter? ¿Cuándo empezaron a publicar tweets sobre el tema y con qué frecuencia?

PI3: ¿Cuál es el origen de los tweets de las dos ONTs durante el primer periodo de crisis de la Covid-19? ¿Fueron de creación propia o retweetearon los de otras organizaciones?

PI4: ¿Qué recursos de comunicación utilizaron las dos ONTs para comunicar contenidos de los tweets durante el primer periodo de crisis de la Covid-19 en Twitter? ¿Fueron recursos gráficos, audiovisuales, o interactivos?

PI5: ¿Qué tipo de reacción en forma de 'me gusta' y comparticiones se generaron entre los públicos a partir de los mensajes publicados por las dos ONTs sobre la crisis de la Covid-19 en Twitter?

Para responder estas preguntas se realizó un análisis de contenido a partir de la creación de una plantilla para examinar los tweets publicados por las dos ONTs, considerando el periodo desde la detección del primer caso de coronavirus Covid-19 identificado en cada país, hasta un mes después que la Organización Mundial de Salud (OMS) declaró el coronavirus como pandemia global (Sevillano, 2020). El 31 de enero se identificó en las Islas Canarias, más específicamente en La Gomera, el "paciente uno" infectado por la Covid-19 en España. Este paciente era de origen alemán y se contagió, presuntamente, al tener contacto con un infectado en Alemania (Arroyo, 2020). Y en Italia, el 21 de febrero, en la ciudad de Codogno, ubicada a 70 kilómetros de Milán, es donde se detectó el primer caso de Covid-19 (Tori, 2020). El paciente era un hombre italiano de 38 años, y a diferencia de España, no se pudo establecer ninguna hipótesis de cómo se produjo el contagio del virus (EFE, 2020).

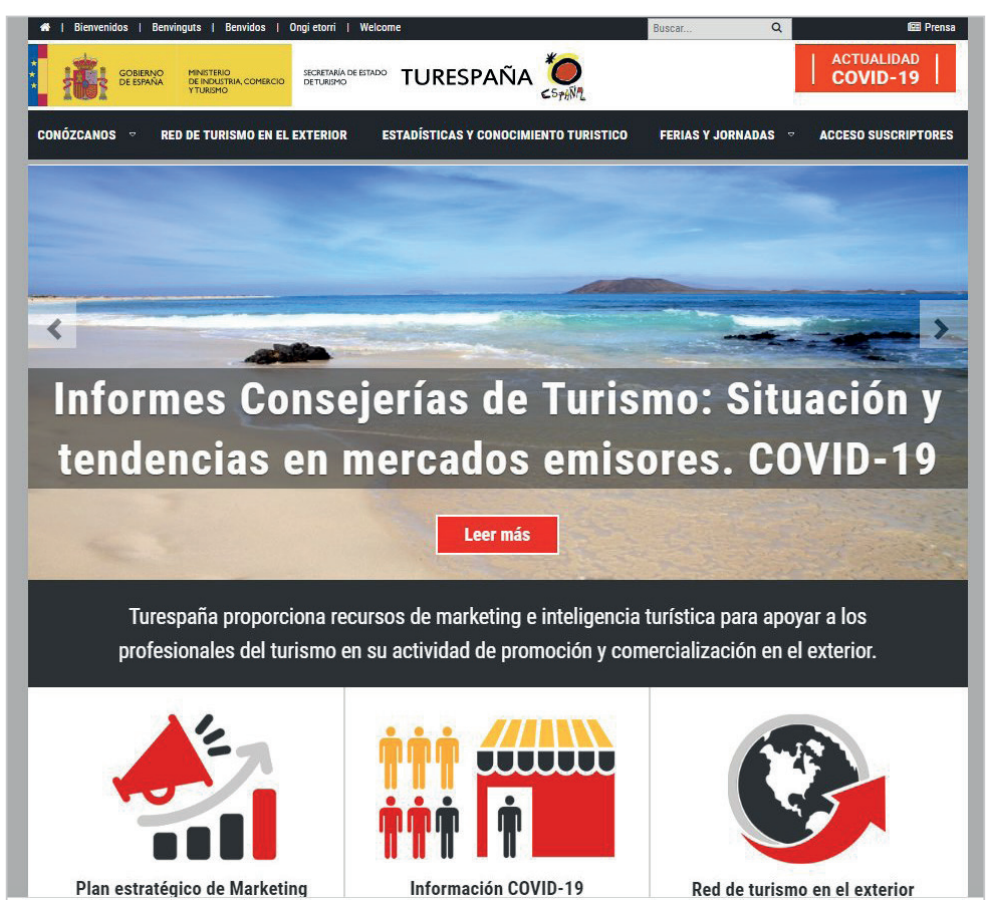

https://www.tourspain.es

Para recopilar los datos se llevó a cabo la recuperación de todos los tweets publicados a través del programa Tuitonomy de las cuentas @Spain y @Italia, filtrando los resultados por fecha de publicación (España del 31 de enero al 11 de abril e Italia del 21 de febrero al 11 de abril). Se obtuvo un total de 519 tweets, siendo 303 de la ONT de España y 216 de la ONT de Italia.

Para el análisis se tuvieron en cuenta los diversos idiomas de las cuentas de Twitter, analizando los tweets en español e inglés de la cuenta de la ONT de España y los tweets en italiano e inglés de la ONT de Italia. Cuando se identificaba duplicación de contenido de tweets en dos idiomas, se optaba por la publicación en inglés.

\subsection{Plantilla de análisis}

La plantilla de análisis de contenido se diseñó partiendo de investigaciones y estudios previos de gestión de crisis y de gestión de crisis a través de Twitter (Cheng, 2020; Coombs, 1998; 2007; 2015; Fowler, 2017;Oliveira; Huertas, 2019; Saffer; Sommerfeldt; Taylor, 2013; Sweetser; Metzgar, 2007; Ulmer; Seeger; Sellnow, 2007; Ulmer; Sellnow; Seeger, 2015; Wang; Zhuang, 2017), adaptándola a las particularidades y características propias de una crisis pandémica y su vinculación con los destinos turísticos. Se prestó especial atención a las informaciones que los países comunican cuando se encuentran en una crisis sanitaria pandémica. Cabe destacar, como señala Xifra (2020, p. 4), que la crisis resultado de la Covid-19, no obedece a la lógica de ningún tipo de crisis anteriormente existente y por lo tanto su gestión no es adaptable a los modelos anteriores de estrategias comunicativas para situaciones de crisis.

De esta forma, y para contestar la PI1, que se centraba en el análisis de contenido, siguiendo a Coombs (1998; 2007; 2015), Sweetser y Metzgar (2007), Ulmer; Seeger y Sellnow (2007), Ulmer; Sellnow y Seeger (2015) y Oliveira y Huertas (2019), la plantilla se estructuró en dos categorías principales:

1) de comunicación sobre la crisis de la Covid-19 y su gestión y

2) de comunicación para salvaguardar la imagen del destino como atracción turística. 
La primera categoría pretendía analizar si se efectuó una correcta comunicación de la crisis de la Covid-19 y de su gestión. Esta categoría englobó dos subcategorías:

1.1) comunicación sobre la crisis de la Covid-19 y

1.2) comunicación sobre gestión de la crisis de la Covid-19 y protección de stakeholders.

La subcategoría 1.1) comunicación sobre la crisis de la Covid-19 comprendía la información general sobre el virus SARSCoV-2, formas y números de contagios, etc., y podría incluir items como: cuándo, dónde, cómo y por qué se propaga el coronavirus o, cómo la crisis de la Covid-19 afecta al turismo debido a posibles cancelaciones de vuelos, de reservas de hoteles, de restaurantes, etc.

En cambio, la subcategoría de 1.2) comunicación sobre gestión de la crisis de la Covid-19 y protección de stakeholders comprendía la información sobre las soluciones que se realizaron para gestionar la crisis de la Covid-19, que podrían englobar:

1.2.1) gestiones en general que no afectaban directamente al sector turístico, explicación sobre el uso de guantes o mascarillas, instrucciones de cómo lavar las manos, frenar rumores, etc.;

1.2.2) gestiones generales que afectaban directamente al sector turístico, como cierre de hoteles, aeropuertos, bares y atracciones turísticas, prohibición de movilidad, ayudas económicas para los agentes del sector, etc., y

1.2.3) gestiones turísticas para afrontar la crisis de la Covid-19, como por ejemplo, información respecto a la seguridad del turista, protocolos de actuación para amparar al turista, exposiciones, conciertos y eventos virtuales como alternativas a las experiencias turísticas o palabras de ánimos.

Por otra parte, la segunda categoría, de gestión estratégica de la comunicación para salvaguardar la imagen del destino, pretendía analizar si el destino realizó comunicación para seguir reforzando su imagen turística mediante sus atractivos. Esta categoría abarcaba comunicación de la promoción turística del destino.

Merece la pena destacar que los tweets analizados se podrían clasificar en más de un tipo de contenido.

Para responder la PI2, en la plantilla de análisis se verificaba la fecha del tweet, puesto que es una información necesaria para valorar la comunicación realizada, dado que como defiende Coombs (1998), la rapidez de la reacción comunicativa es un factor clave de éxito en una situación de crisis.

Para responder la PI3, la plantilla de análisis contemplaba el origen del mensaje, distinguiendo entre tweets propios y retweets de otras organizaciones. Ello es fundamental para analizar la implicación que las ONTs examinadas tuvieron en la comunicación de la crisis de la Covid-19, debido a la importancia de que asumieran un rol activo en la comunicación como fuente primaria de información durante una crisis que afecta directamente al sector turístico (Fowler, 2017; Granville; Mehta; Pike, 2016).

Para responder la PI4 se contemplaron en la plantilla de análisis los recursos de comunicación que las ONTs utilizaron para difundir los mensajes a partir de las categorías del estudio desarrollado por Capriotti, Zeler y Oliveira (2019), en el cual los autores señalan que los videos son el formato que más engagement genera entre los turistas. Así, se distinguieron tres tipos de recursos o medios comunicativos:

- gráficos (texto, imagen, emoticono),

- audiovisuales (imagen animada, audio-video), e

- interactivos (link, hashtag, etiqueta a usuarios).

Tabla 1. Plantilla de análisis de contenido

\begin{tabular}{|c|c|c|}
\hline \multicolumn{3}{|l|}{ Fecha } \\
\hline Origen & $\begin{array}{l}\text { Tweet } \\
\text { Retweet (¿De quién?) }\end{array}$ & \\
\hline Categorías de análisis & Subcategorías & \\
\hline \multirow{2}{*}{$\begin{array}{l}\text { 1) Comunicación sobre la } \\
\text { crisis de la Covid-19 y su } \\
\text { gestión }\end{array}$} & \multicolumn{2}{|c|}{ 1.1) Comunicación sobre la crisis de la Covid-19 } \\
\hline & $\begin{array}{l}\text { 1.2) Comunicación sobre gestión de } \\
\text { la crisis de la Covid-19 y protección } \\
\text { de stakeholders }\end{array}$ & $\begin{array}{l}\text { 1.2.1) Gestiones en general que no afectaban directamente al sector turístico. } \\
\text { 1.2.2) Gestiones generales que afectaban directamente al sector turístico. } \\
\text { 1.2.3) Gestiones turísticas para afrontar la crisis de la Covid-19. }\end{array}$ \\
\hline \multicolumn{3}{|c|}{ 2) Comunicación para salvaguardar la imagen del destino como atracción turística } \\
\hline Recursos de comunicación & & $\begin{array}{l}\text { Gráficos (texto, imagen, emoticón) } \\
\text { Audiovisuales (imagen animada, audio-video) } \\
\text { Interactivos (link, hashtag, etiqueta a usuarios) }\end{array}$ \\
\hline Nivel de engagement & $\begin{array}{l}\text { No de me gusta } \\
\text { No de comparticiones }\end{array}$ & \\
\hline
\end{tabular}


Por último, para contestar la PI5, la plantilla pretendía valorar las reacciones de los usuarios a los tweets, a través del análisis del nivel de engagement de los seguidores, que englobaba la contabilidad de los 'me gusta' y comparticiones recibidos por los tweets. Estas mediciones contribuyen a valorar el éxito de las estrategias comunicativas aplicadas por las ONTs (Cheng, 2020; Coombs, 2007; Saffer; Sommerfeldt; Taylor, 2013; Sweetser; Metzgar, 2007; Uşaklı; Koç; Sönmez, 2017).

El análisis de contenido de los tweets a través de la plantilla (tabla 1) se realizó de forma manual durante el mes de abril de 2020.

\section{Resultados}

\subsection{Tipos y contenidos de los tweets publicados}

Si analizamos los tipos de contenidos, en respuesta a la PI1, se observa que ninguna de las ONTs publicó los contenidos relacionados con la categoría de comunicación sobre la crisis de la Covid-19 en Twitter. No obstante, sí que se detecta un esfuerzo por parte de las ONTs en realizar una comunicación sobre la gestión de la crisis de la Covid-19 y protección de stakeholders. Dentro de la totalidad de los tweets publicados, casi una cuarta parte (23,5\%) de los mensajes fueron de este tipo, destacando la subcategoría: comunicación de gestiones turísticas para afrontar la crisis de la Covid-19 (con $21,4 \%$ de los tweets).

De manera específica, la ONT de Italia dedicó poco más de la mitad de sus tweets (el 53,2 \%) a información para gestionar la crisis y proteger a los stakeholders. En este sentido, la ONT de Italia se comunicó de dos formas. Por un lado, mayoritariamente comunicó gestiones turísticas para afrontar la crisis de la Covid-19 (el 50,5 \% de sus tweets). Para este tipo de información, esta ONT publicó contenido difundiendo que Italia era un país seguro y destacaba su reputación como destino de calidad, informando de la normalidad de los servicios ofrecidos a los ciudadanos y turistas. Cabe destacar que para ese tipo de tweet ofrecía un enlace:

http://www.italia.it/es/informacion-util/covid-19-informacion-y-actualizaciones-para-los-turistas.html

por si el seguidor quisiera más información sobre el Coronavirus, que llevaba a un apartado de la Agenzia Nazionale Italiana del Turismo denominado "Covid-19 - Información y actualizaciones para los turistas", y contenía información actualizada sobre la Covid-19 en diversos idiomas. Asimismo, la ONT de Italia publicó mensajes con la finalidad de ofrecer experiencias turísticas alternativas con fotos, conciertos, exposiciones virtuales a sus seguidores que perseguía mostrar las bellezas italianas a todos los hogares del mundo mediante el empleo de hashtags como \#BellezzaAdomicilio y \#italystaystrong (Italia se mantiene fuerte), respectivamente. El contenido dentro de esta categoría impulsaba comunicaciones de apoyo con el objetivo de hacer más liviano el periodo del confinamiento, pero a la vez recordaba a los públicos sobre los muchos atractivos del destino Italia.

Algunos ejemplos de tweets son:

@italia tweeted FEB 28 2020: "Italy is a safe country: all services and activities for citizens and tourists are normally provided and the quality of life, for which \#Italy is famous world-wide, remains high". More info http://bit.ly/italy-a-safe-country-covid-19...\#COVID19

@italia tweeted MAR 112020 "The beauty of \#Italy comes to you! We share our wonders with you every day even if you can not be here now. Enjoy this amazing view of \#Florence"... and find updated infos from Italy here http://bit.ly/COVID19-Info_and_updates_for_tourists...\#Treasureltaly \#italystaystrong

Por otro lado, y de manera muy minoritaria, la ONT de Italia también comunicó sobre cuestiones generales para afrontar la crisis de la Covid-19 que afectaban directamente al sector turístico (2,8\% de sus tweets). En este tipo, la ONT de Italia difundió contenidos que invitaban a los turistas y residentes a quedarse en casa y a mantenerse seguros. Estos tweets recordaban la importancia de apoyar a las políticas de seguridad mediante el confinamiento que impactan negativamente en el sector turístico. Aquí se presentan ejemplos en los que la ONT de Italia empleó el hashtag quedarse en casa, y recordó la imposibilidad de poder viajar:

@italia tweeted MAR 24 2020: "In attesa di poter tornare a viaggiare, le bellezze della Lombardia possono essere ammirate anche da casa. Seguiamo i canali @inLOMBARDIA: nei prossimi giorni tante novità!" \#iorestoacasa \#BellezzaAdomicilio \#inLombardia

@italia ABR 7 2020: "Let us throw an ideal coin in the Trevi Fountain, wishing you all will be able to come back soon! In the meantime, stay safe and enjoy this amazing video from home \#Italycomestoyou \#Treasureltaly \#Rome @Turismoromaweb@visit_lazio) 


\begin{tabular}{|c|c|c|c|c|c|c|}
\hline & \multicolumn{2}{|c|}{ General } & \multicolumn{2}{|c|}{ España } & \multicolumn{2}{|c|}{ Italia } \\
\hline & $\mathbf{n}$ & $\%$ & $\mathbf{n}$ & $\%$ & $\mathbf{n}$ & $\%$ \\
\hline \multicolumn{7}{|l|}{ 1. Comunicación sobre la crisis de la Covid-19 y su gestión } \\
\hline 1.1. Comunicación sobre la crisis de la Covid-19 & 0 & 0,0 & 0 & 0,0 & 0 & 0,0 \\
\hline $\begin{array}{l}\text { 1.2. Comunicación sobre la gestión de la crisis de la Covid-19 y protección de } \\
\text { stakeholders }\end{array}$ & 122 & 23,5 & 7 & 2,3 & 115 & 53,2 \\
\hline 1.2.1. Gestiones en general que no afectaban directamente al sector turístico & 0 & 0,0 & 0 & 0,0 & 0 & 0,0 \\
\hline 1.2.2. Gestiones generales que afectaban directamente el sector turístico & 11 & 2,1 & 5 & 1,7 & 6 & 2,8 \\
\hline 1.2.3. Gestiones turísticas para afrontar la crisis de la Covid-19 & 111 & 21,4 & 2 & 0,7 & 109 & 50,5 \\
\hline 2. Comunicación para salvaguardar la imagen del destino como atracción turística & 503 & 96,9 & 302 & 99,7 & 201 & 93,1 \\
\hline Total & 519 & & 303 & & 216 & \\
\hline
\end{tabular}

Por el contrario, la ONT de España comunicó mucho menos estos contenidos si se compara con la ONT de Italia, porque sólo difundió 2 tweets (el $0,7 \%$ ) con contenidos sobre las gestiones turísticas para afrontar la crisis de la Covid-19. Su estrategia se limitó a compartir tweets de otras entidades, como por ejemplo el de la oficina de turismo de Jaén, que fomentaba a los seguidores nacionales y futuros turistas en situación de confinamiento a realizar una experiencia turística desde el hogar:

@spainretweeted@jaenturismo MAR 14 2020: “Seguimos viajando \#desdecasaHousebuildings. Os dejamos esta espectacular imagen de la cascada de la \#Cimbarra. Es uno de los parajes naturales más impresionantes de \#JaenParaisolnterior. https://bit.ly/33gwRXL @dipujaen @ spain \#aldeaquemada \#viajardesdecasa \#YoMeQuedoEnCasa https://pic.Twitter.com/vdlmmZDOrl

En la misma línea, la ONT de España casi no difundió información sobre las gestiones para afrontar la crisis de la Covid-19 que afectaban directamente al sector turístico (con solo el 1,7 \% de sus tweets). De manera concreta, la ONT de España publicó un tweet que mencionaba directamente la pandemia mediante el uso del \#Covid19 en el que informaba que España estaba luchando contra el Coronavirus, solicitando a sus seguidores que se cuidasen, y a la vez invitando a una visita futura al destino. El tweet no incluye enlace para ampliar o actualizar información sobre Covid-19

@spain tweeted Mar 18 2020: "Spain is fighting with all its strength against \#COVID19. We are a great country, and very soon we will be able to welcome you again with open arms and our characteristic hospitality. Until then, take care of yourselves and others around you. Thanks for the support"

El otro tipo de contenido que la ONT de España publicó, al igual que la de Italia, incentivaba a los seguidores a quedarse en casa, aunque consecuentemente, ello perjudicaba al sector turístico

@spainretwitted Mar 142020 @CyLesVida “QQUÉDATE EN CASA! ¡Seamos responsables! Naturaleza, cultura, patrimonio, arte, museos, tradiciones... Para poder disfrutarlo luego, ahora... ¡Quédate en casa!" \#yomequedoencasa https://pic.twitter.com/FshTzOTYbg

Cabe destacar que ninguna de las dos ONTs publicó tweets con información de gestiones generales sobre la Covid-19 que no afectaban directamente al sector turístico.

Por último, si se observa la categoría comunicación para salvaguardar la imagen del destino como atracción turística, se reconoce que ambas ONTs exploraron de manera contundente este tipo de contenido en la plataforma (más de $90 \%$ de la totalidad de tweets). De manera específica, la ONT de Italia publicó el 93,1\% de sus tweets en esta categoría para explotar sus fortalezas turísticas. Los tweets se centraban en facilitar fotografías y datos históricos variados sobre edificios emblemáticos como la torre de Pisa, el castillo de Ischia o la Cappella degli Scrovegni (figura 1). Asimismo, se identifica que la ONT de Italia difundió mensajes sobre celebraciones y recordatorios relacionados con los pintores italianos, como es el caso de la celebración del aniversario de la creación del cuadro Venus pintado por Botticelli, y poetas italianos y actividades culturales como las exhibiciones de los museos. También publicó tweets sobre la gastronomía del país, cómo cocinar pasta y postres típicos italianos y mensajes que promocionaban diferentes regiones y ciudades italianas como Verona, Florencia o Bolonia.

De forma similar, la ONT de España difundió un alto volumen de contenido para salvaguardar la imagen del destino como atracción turística (casi el $100 \%$ de sus tweets). De hecho, durante el periodo analizado, el organismo español prácticamente se limitó a proyectar sus encantos turísticos mediante una variedad de contenidos, como

La Agenzia Nazionale Italiana del Turismo fue más rápida y activa al inicio de la crisis 


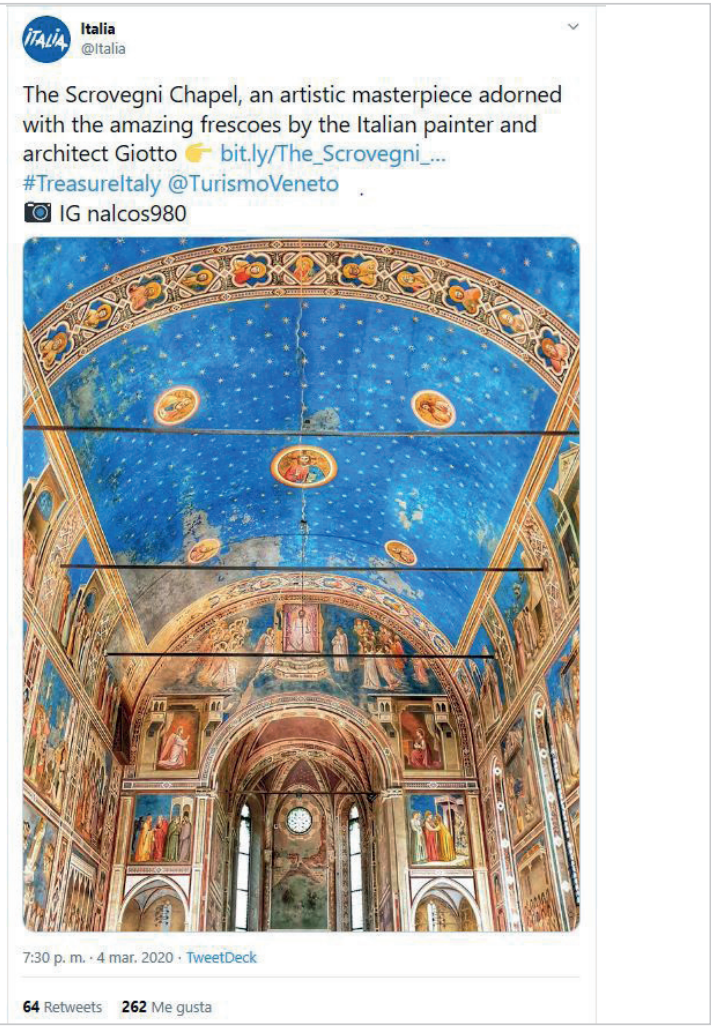

Figura 1. Contenido de promoción turística de la ONT italiana. https://twitter.com/Italia/status/1235271290010750976
“' Spain @ @

Mientras esperamos a la \#Primavera... \#Soria nos regala esta bella estampa $\bigcirc$. Si te gusta el senderismo, tienes una infinidad de rutas para disfrutar a lo grande de sus paisajes. ¿Te animas a descubrirla?

\#VisitSpain \#SpainNature @Soriaestademoda @sorianitelaimag@CyLesVida
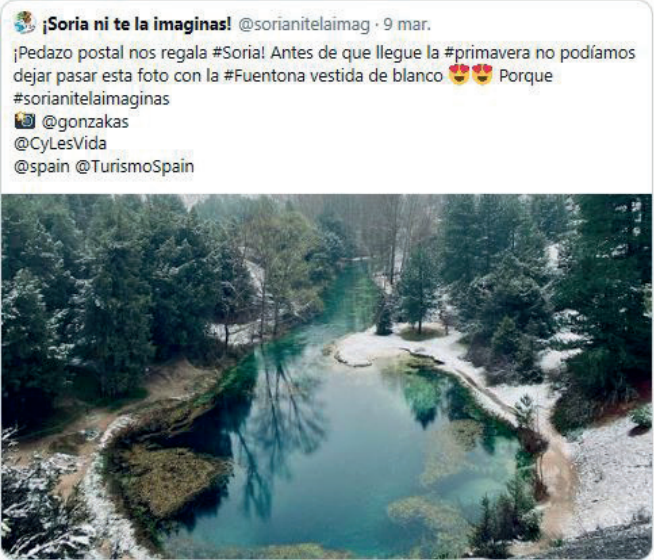

9:08 p. m. 9 mar. 2020 - Twitter Web App

13 Retweets 94 Me gusta

Figura 2. Contenido de promoción turística de la ONT española. https://twitter.com/spain/status/1237108122294136833

platos típicos de ciudades españolas, planes sobre qué hacer en algunas ciudades, recopilación de leyendas o historias de diferentes lugares, guía de los miradores más impresionantes de España, información sobre servicios de transporte para poder viajar, información sobre el mar Mediterráneo o sobre el entorno natural de España (figura 2).

\subsection{Timing de la publicación de los tweets}

El análisis del timing (PI2) muestra que las ONTs publicaron tweets a ritmos distintos a partir de la detección del primer paciente en cada país. En el caso de España, el primer caso positivo apareció el día 31 de enero, sin embargo, el primer tweet que la ONT de España publicó al respecto no apareció hasta 43 días después. Lo hizo mediante un retweet de la Oficina de Turismo de Castilla y León, en el que se aludía a la crisis de manera indirecta solicitando a los seguidores a afrontar la situación con responsabilidad, evitando la movilidad y practicando el confinamiento (figura 3).

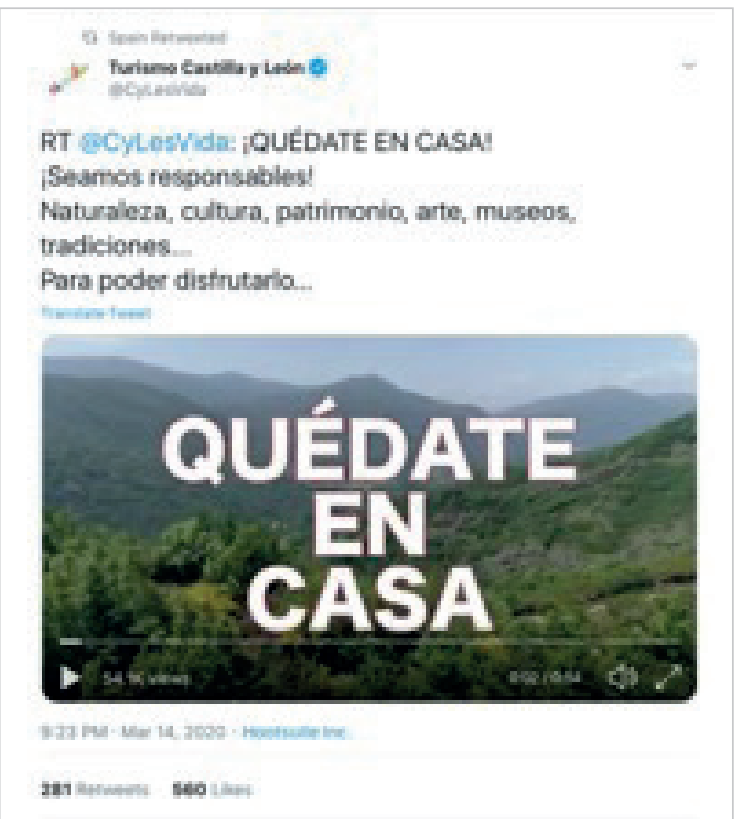

Figura 3. Primera comunicación de la ONT española vinculada a la crisis de la Covid-19.

https://twitter.com/spain/status/1239254521739644928

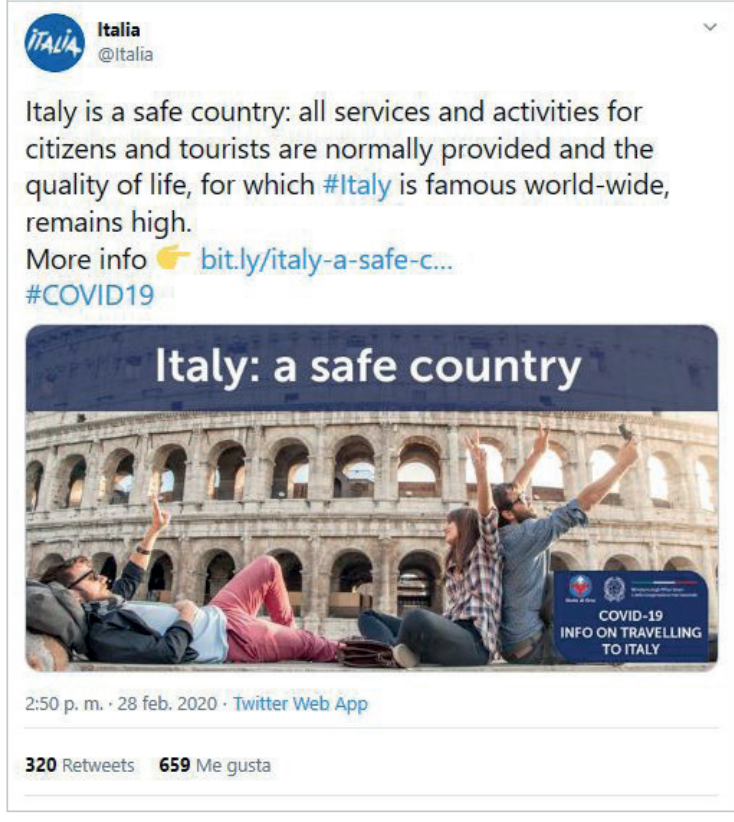

Figura 4. Primer tweet de la ONT italiana sobre Covid-19. https://twitter.com/Italia/status/1233389065724186624 


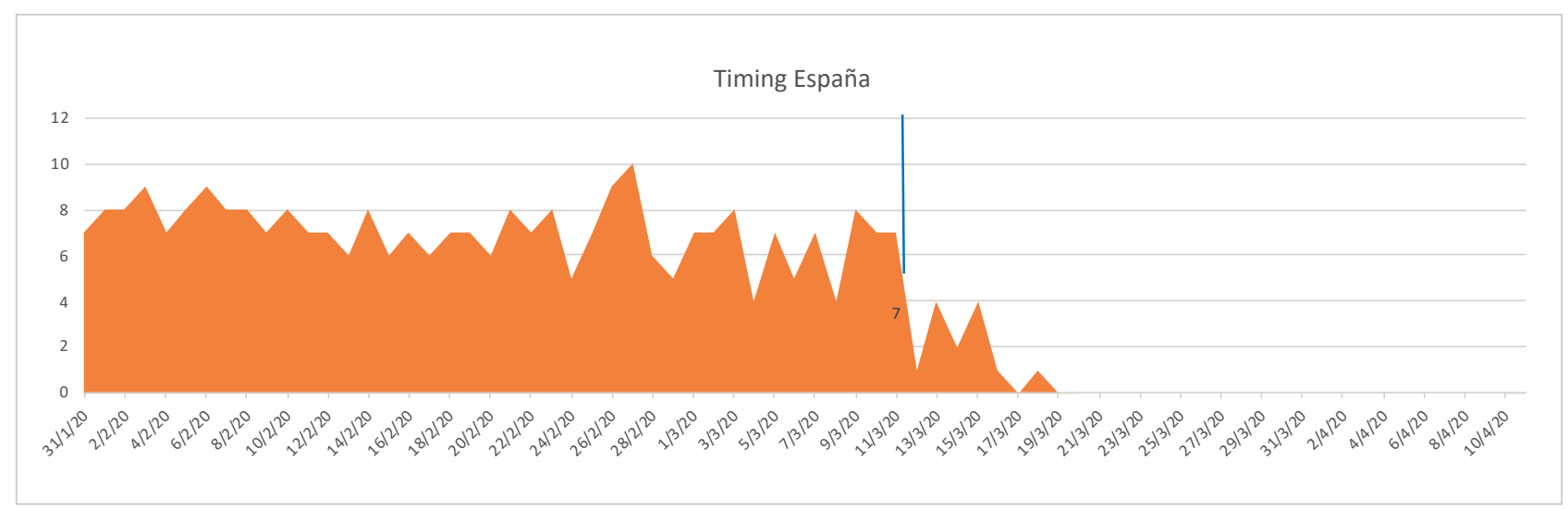

Grafico 1. Cantidad de tweets publicados por la ONT española

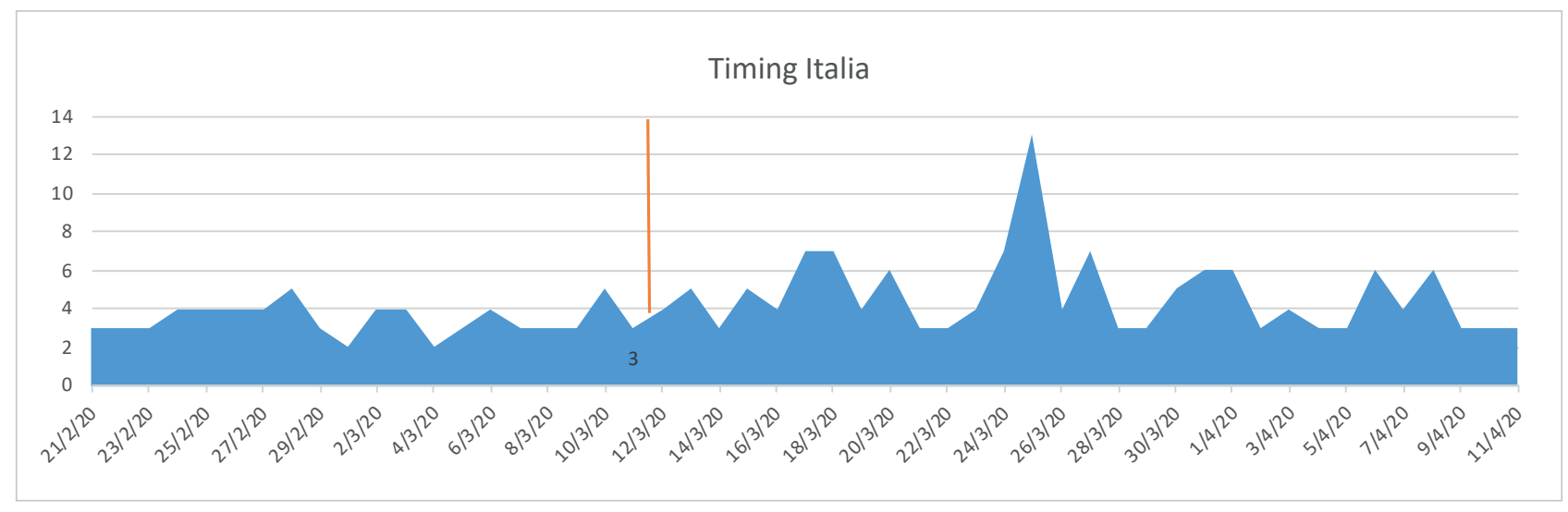

Grafico 2. Cantidad de tweets publicados por la ONT italiana

En el caso de Italia, el primer paciente fue diagnosticado el día 21 de febrero, y la ONT de Italia publicó el primer tweet directamente vinculado a este tema 8 días después. El tweet de la ONT destacaba que Italia era un país seguro y ofrecía un enlace para que el turista se informase sobre las actualizaciones relacionadas con la Covid-19 (figura 4).

Si se compara el ritmo en que las ONTs publicaron, teniendo como referencia temporal el espacio entre la identificación del primer paciente y la declaración oficial de la pandemia por la OMS (gráficos 1 y 2), se observa que la ONT de España fue más activa en la plataforma mayoritariamente antes de la declaración, dado que publicó el 93,4\% de la totalidad de sus tweets en este período, mientras que la ONT de Italia concentró alrededor de una tercera parte de sus publicaciones (30,6\% de la totalidad de sus tweets) en la misma etapa. Es importante destacar que el periodo de días entre la identificación del primer paciente y la declaración de la pandemia es mayor en España, con lo cual la ONT española tuvo más tiempo para preparar la gestión de su comunicación.

Cabe resaltar que la ONT de Italia mencionó la Covid-19 antes de la declaración de la pandemia por parte de la OMS el día 11 de marzo. Específicamente, el organismo italiano publicó 2 tweets que aludían directa o indirectamente al Covid-19 antes de la declaración de la pandemia y 130 tweets después. En cambio, la ONT de España solo mencionó el tema después de la declaración de la pandemia. Concretamente lo hizo mediante un tweet y 4 retweets. Se puede observar que la institución española ha sido mucho más lenta y pasiva respecto a la información sobre la crisis de la Covid-19.

Cabe destacar que en el marco temporal posterior a la declaración oficial de la pandemia por la OMS, se detecta que la ONT de España publicó 20 tweets (el 6,6\% de la totalidad de sus tweets); y a partir del día 18 de marzo, siete días después de la oficialización de la pandemia, dejó de difundir contenido en su cuenta, es decir, se mantuvo en silencio durante el resto del periodo analizado, lo que resulta sorprendente. En este sentido, la ONT de España publicó 7 tweets el día de la declaración, pasando a 1 el día siguiente, con una variación de 5 o 2 diarios (donde 4 fue la mayor cantidad de tweets publicados en un único día, el 13 de marzo). En contraste, Italia, tras la declaración, mantuvo una difusión activa $(69,44 \%$ de la totalidad de sus tweets), con un pico de volumen el día 25 de marzo con 13 tweets publicados. Específicamente, la ONT de Italia sólo publicó 3 tweets el día de la declaración, pero su actividad se mantuvo constante, con una variación promedio de 4,7 tweets diarios.

Este estudio contribuye a dar pautas de comunicación a las ONTs durante crisis sanitarias 
La ONT de Italia reaccionó con bastante rapidez, publicando un tweet que hacía referencia al suceso; a diferencia de la ONT de España, que no difundió ningún contenido sobre los hechos hasta bastante después. De otra parte, si tenemos en cuenta el periodo posterior a la declaración de la pandemia, la organización italiana fue mucho más activa que la española en la gestión de su comunicación en Twitter manteniendo y aumentando su actividad. Esta diferencia se evidencia todavía más si se considera el promedio total de tweets publicados por ambas ONTs después de la declaración de la OMS: la ONT de Italia $(4,7)$, mientras que la ONT de España $(0,6)$.

\subsection{Origen de los tweets}

En relación con el origen del mensaje (PI3), se observa en la tabla 3 que poco más del $90 \%$ de los tweets publicados por ambas ONTs fueron tweets de producción propia.

Tabla 3. Origen de los mensajes publicados

\begin{tabular}{|c|c|c|c|c|c|c|}
\hline & \multicolumn{2}{|c|}{ General } & \multicolumn{2}{|c|}{ España } & \multicolumn{2}{|c|}{ Italia } \\
\hline & n & $\%$ & n & $\%$ & $\mathbf{n}$ & $\%$ \\
\hline Tweet & 474 & 91,3 & 278 & 91,7 & 196 & 90,7 \\
\hline Retweet & 45 & 8,7 & 25 & 8,3 & 20 & 9,3 \\
\hline Total & 519 & 100 & 303 & 100 & 216 & 100 \\
\hline
\end{tabular}

Cabe destacar que el origen de las 5 únicas publicaciones que la ONT de España difundió en su plataforma que aludía al Covid-19, una era de origen propio (figura 5) y las 4 restantes fueron retweets (figura 3). Estos retweets provenían de entidades de turismo local, concretamente de las cuentas @Soriaestademoda, @jaenturismo, @CyLesVida (Turismo Castilla y León) y @CaminosGuadiana. Casi todos los retweets incluían el \#YoMeQuedoEnCasa.

En cambio, en la ONT italiana podemos observar que los tweets propios que mencionaron la crisis de la Covid-19 fueron 132 y todos los retweets excepto uno aludieron a la crisis. Los retweets provenían de cuentas de oficinas de turismo de regiones italianas:

@VisitSicilyOP, @YourAbruzzo, @VisitTrentino, @TurismoVeneto, @UmbriaTourism, @TurismoLiguria, o bien de entidades culturales como @MuseoArcheoCa y @Museo_MAXXI.

Las cuentas de las regiones de Veneto y Sicilia destacaron hashtags específicos como \#RegaliamociBellezza y \#sicilyathome. Todos los retweets tuvieron en común el uso de alguno de estos hashtags \#BellezzaADomicilio o \#Italycomestoyou, así como \#iorestoacasa.

\subsection{Recursos de comunicación}

Respecto a la (PI4), los resultados muestran que ambos países presentan similitudes en referencia al uso de recursos de comunicación.

Tabla 4. Recursos de comunicación utilizados por las ONTs en Twitter

\begin{tabular}{|c|c|c|c|c|c|c|}
\hline \multirow{2}{*}{ Recursos de comunicación } & \multicolumn{2}{|c|}{ General } & \multicolumn{2}{|c|}{ España } & \multicolumn{2}{|c|}{ Italia } \\
\hline & $\mathbf{n}$ & $\%$ & $\mathbf{n}$ & $\%$ & $\mathbf{n}$ & $\%$ \\
\hline Recursos gráficos & 494 & 95,2 & 289 & 95,4 & 205 & 94,9 \\
\hline Recursos interactivos & 519 & 100 & 303 & 100 & 216 & 100 \\
\hline Recursos audiovisuales & 26 & 5,0 & 14 & 5 & 12 & 5,6 \\
\hline Total & 519 & & 303 & & 216 & \\
\hline
\end{tabular}

Los recursos interactivos y gráficos (figura 6) son los más utilizados por ambas ONTs para difundir los contenidos en Twitter (en más del $90 \%$ de los tweets). En los recursos gráficos predomina el texto con foto y en los recursos interactivos prevalecen los hashtags y los links. Por otra parte, se identifica que los recursos audiovisuales (solo en el $5 \%$ de los tweets), son los que las ONTs utilizan menos para comunicar los contenidos en la plataforma. 


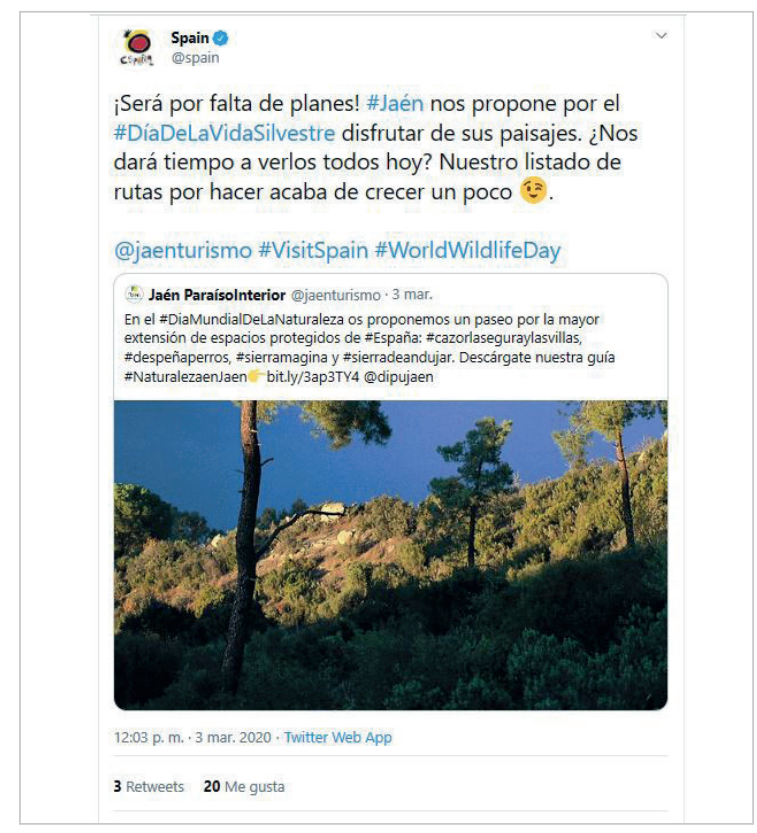

Figura 6. Ejemplo de formato gráfico de ONT España.

https://twitter.com/spain/status/1234796416570142721

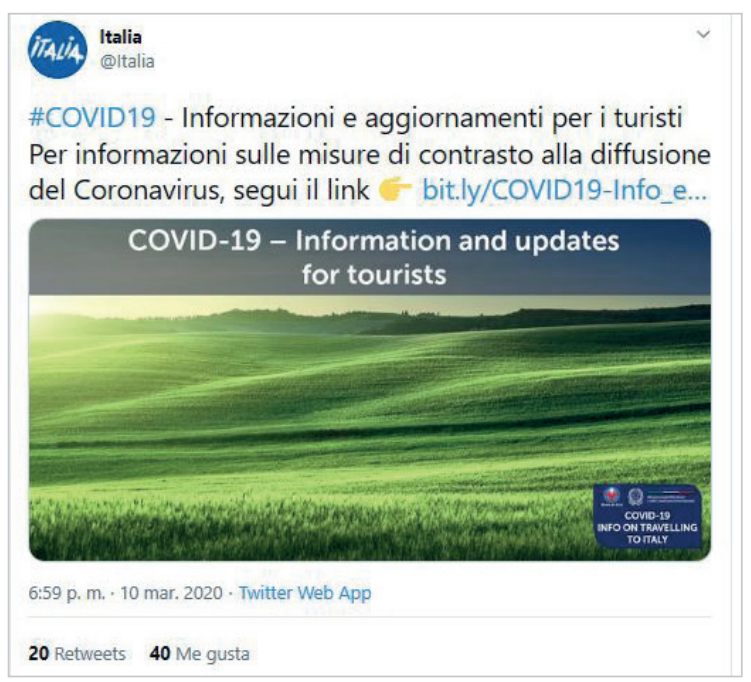

Figura 7. Tweet de la ONT italiana con \#COVID19. https://twitter.com/italia/status/1237437935399710721

Si se consideran los recursos como los hashtags y las etiquetas, los que la ONT España más utilizó fueron \#visitspain, \#livespain, \#spainnature, \#spaingastro, \#spainexperience y \#ingreenspain, que aluden a contenidos de promoción turística. En contrapartida, utilizó una única vez \#COVID19, \#desdecasa y \#yomequedoencasa, que son los hashtags más vinculados directamente con la crisis (figura 5).

Asimismo, se identifica que los usuarios que la ONT más mencionó fueron @viveandalucia; @CyLesVida, @Turgalicia, @ turismoclm y @Extremadura_tur.

En el caso de Italia, se detecta que dos de los tres hashtags que la ONT más empleó están directamente vinculados a la crisis de la Covid-19: \#treasureitaly (142 veces), \#Bellezzadomicilio (78 veces) y \#italycomestoyou (63 veces).

De hecho, después de la declaración del estado de emergencia el 11 de marzo, todos los tweets que la ONT de Italia publicó incluyeron uno de los siguientes hashtags en su texto: \#italycomestoyou, \#Bellezzadomicilio o \#iorestoacasa. Merece la pena destacar que la ONT de Italia utilizó \#COVID19 dos veces en el periodo analizado (figuras 4 y 7). Respecto a los usuarios que más aludió son: @VisitTuscany; @BorghiPiuBelli; @VisitSicilyOP; @visit_lazio; @TurismoVeneto@ Basilicata_Tur; @Turismoromaweb.

\subsection{Reacciones de los tweets publicados}

Respecto a la PI5, los resultados obtenidos (tabla 5) indican que el nivel de engagement total se materializó en 65.340 'me gusta', y 16.716 comparticiones de los seguidores. Los mensajes de la ONT de España obtuvieron 24.326 'me gusta', lo que supone una media de 80,28 'me gusta' por tweet, mientras que los tweets de la ONT de Italia recibieron en total 41.014 'me gusta', con una media de 189,00 por publicación. En este sentido, cabe destacar que la ONT de Italia acumuló casi el $63 \%$ de la totalidad de los 'me gusta' generados respecto a la de España, en un período menor de tiempo. En la misma línea, los tweets publicados por la ONT de España generaron 6.748 comparticiones, con una media de 22,27 por tweet, mientras que la ONT italiana obtuvo un total de 9.968 comparticiones, con una media de 45,94 por publicación.

Tabla 5. Reacciones generadas entre los públicos

\begin{tabular}{|c|c|c|c|c|}
\hline & ‘Me gusta' & Comparticiones & $\begin{array}{c}\text { Media de 'me gusta' por } \\
\text { tweet }\end{array}$ & $\begin{array}{l}\text { Media de comparticiones } \\
\text { por tweet }\end{array}$ \\
\hline ONT España & 24.326 & 6.748 & 80,28 & 22,27 \\
\hline ONT Italia & 41.014 & 9.968 & 189,00 & 45,94 \\
\hline Total & 65.340 & 16.716 & & \\
\hline
\end{tabular}

Cabe destacar que los tweets que las ONTs publicaron que tuvieron más apoyo y comparticiones están relacionados con la crisis de la Covid-19. El tweet de la ONT de España que recibió más interacciones (367 comparticiones y más de 1.616 'me gusta') fue: 
@spain tweeted Mar 18 2020: "Spain is fighting with all its strength against \#COVID19. We are a great country, and very soon we will be able to welcome you again with open arms and our characteristic hospitality. Until then, take care of yourselves and others around you. Thanks for the support"

En cambio, el segundo tweet por el que la ONT de España recibió más interacciones (201 comparticiones y 608 'me gusta'), aunque con menor nivel de engagement, tenía un contenido de promoción turística. Concretamente era una pieza audiovisual que celebraba el día de Andalucía e invitaba a explorar sus 8 provincias:

@spain tweeted FEB 28 2020: "Today is Andalucía's day! We invite you to explore with us its 8 unique provinces. Have you visited any of them? bit.ly/32eZW5b \#VisitSpain \#Andalucía \#ViveAndalucía \#DíaDeAndalucía @viveandaluciahttps://t.co/1DakQEzKcP"

Así pues, cabe destacar que el tweet que fue compartido más veces y que obtuvo más 'me gusta', fue la única publicación creada y difundida por el organismo sobre la crisis de la Covid-19 en formato "texto", lo que sugiere que, pese a no ser el tipo de recurso de comunicación que genera más engagement entre los usuarios, el tema de la Covid-19 fue el que despertó más implicación, interés, apoyo y participación de los seguidores en el periodo analizado.

En el caso de la ONT de Italia se observa que los dos tweets con mayor nivel de engagement están vinculados con la Covid-19. El tweet, que más 'me gusta' (1432) y más comparticiones (481) acumuló durante el periodo analizado (figura 8), incluía una pieza audiovisual que mostraba diferentes italianos cantando la canción "Azurro", y contenía un mensaje de agradecimiento a todos los usuarios que habían compartido sus videos caseros e incluía dos de los hashtags más usados en las publicaciones \#treasureitaly (142) y \#italycomestoyou (63).

En el segundo tweet con mayor número de 'me gusta' ( $n=992$ ) entre los seguidores, la ONT de Italia lanza simbólicamente una moneda en la Fontana de Trevi expresando el deseo que los turistas puedan regresar cuanto antes, solicita que los usuarios cuiden de su salud respetando el confinamiento y ofrece una pieza audiovisual con espectaculares imágenes para suavizar el confinamiento:

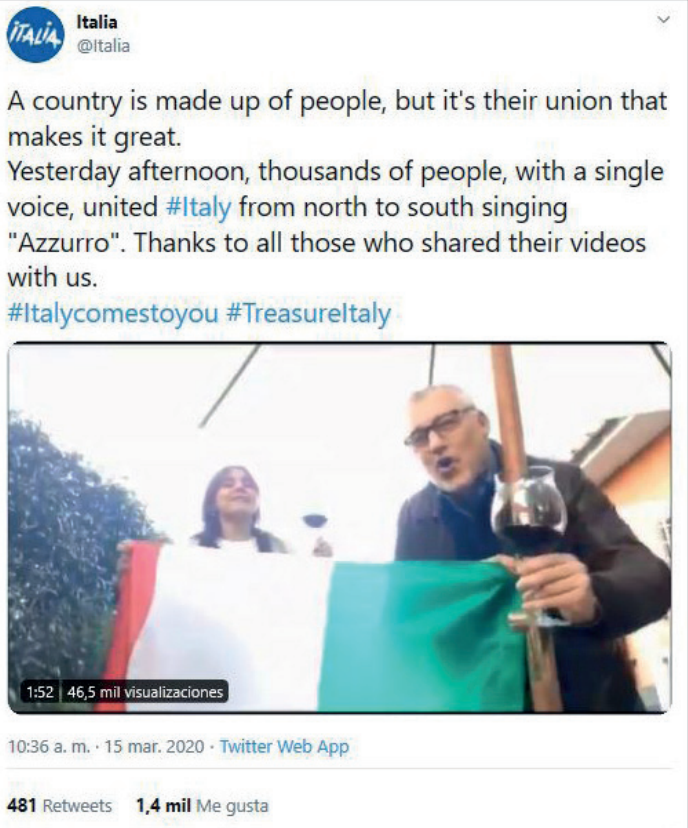

Figura 8. Tweet de ONT italiana más apoyado y compartido por los seguidores.

https://twitter.com/italia/status/1239123413836410881

@italiatweeted ABR 7 2020: "Let is throw an ideal coin in the Trevi Fountain, wishing you all will be able to come back son! In the mean time, stay sate and enjoy this amazing video from home \#italycomestoyou \#Treasureltaly \#Rome @Turismoromaweb @visit_lazio

El segundo tweet de la ONT de Italia con más comparticiones entre los seguidores (320), hace referencia a la primera publicación que la ONT difundió sobre la Covid-19 informando que Italia era un país seguro. En él se incluía una foto de turistas en el Coliseo y un enlace de la Agenzia Nazionale Italiana del Turismo con información y actualización para el turista sobre la Covid-19 (figura 4).

\section{Discusiones y conclusión}

En primer lugar, el estudio demuestra las grandes diferencias de contenido existentes en los tweets de las dos ONTs analizadas. Mientras que la italiana publicó la mitad de sus tweets para informar sobre las gestiones turísticas que realizaron para afrontar la crisis de la Covid-19 (109 tweets), los tweets sobre este tema fueron prácticamente inexistentes en la cuenta de la ONT española (tan solo 2 tweets). Así pues, parece que la ONT de Italia informó y estuvo al lado del turista aportando información logística y mostrando experiencias turísticas virtuales en tiempo de confinamiento; mientras que la ONT de España no informó suficientemente del tema. Y ello es de fundamental importancia en una crisis sanitaria de este tipo, ya que los turistas evitan el riesgo y quieren sentirse seguros (Araña et al., 2009; Reisinger; Mavondo, 2005). Para ellos es importante saber que las organizaciones como las ONTs se movilizarán y les ayudarán en situaciones de emergencia, porque no solo hablamos de clientes, sino también de públicos (Xifra, 2020). Por otro lado, la ONT de España publicó más tweets turísticos para salvaguardar la imagen del destino. Ello va en la línea de los comentarios de la ministra española sobre la estrategia de reposicionar la marca España como un destino seguro (Sérvulo Gonzalez; Salvaterra, 2020). La ministra informó sobre los protocolos de actuación que habían llevado a cabo, pero todo ese esfuerzo de acciones realizadas no se comunicó a través del Twitter de la ONT de España. 
Además, la ONT de España publicó el primer tweet sobre la Covid-19 43 días después del primer infectado en el país y dejó de publicar tweets quedando en silencio durante los 7 días siguientes a la declaración de la pandemia; mientras que la de Italia comenzó a publicar tan solo 8 días después manteniendo sus publicaciones constantes durante todo el periodo. Así pues, se observa que la ONT española fue lenta en la comunicación de la crisis y poco estratégica al dejar de publicar contenidos con posterioridad a la declaración de la pandemia, ya que los usuarios esperan informaciones continuadas, especialmente en momentos de incertidumbre generados por este tipo de crisis sanitarias (Gui et al., 2017).

Por otro lado, el estudio también demuestra que los tweets de la ONT italiana obtuvieron el doble de reacciones por parte de los usuarios que los de la ONT española, tanto en los me gusta como en las comparticiones. Y ello se debe a que publicaron más tweets sobre la gestión que realizaron por la Covid-19. De hecho, los tweets que obtuvieron más reacciones fueron especialmente los de la Covid-19. Así pues, el estudio demuestra que a los turistas o usuarios les gusta y comparten más los tweets que muestran una gestión de la entidad ante la crisis sanitaria de la Covid-19. Ello va en la línea de lo que recomendaron Boin y Lagadec (2000) previamente, respecto a la importancia que las organizaciones informen y se hagan presentes en los momentos de crisis ante los públicos, que en esos momentos se sienten confundidos (Marco; López, 2010). Por otro lado, las reacciones de los usuarios muestran el éxito de las publicaciones realizadas por las ONTs; y, además, con los 'me gusta' y las comparticiones los propios usuarios están fomentando la difusión y la viralidad de estos contenidos (Colley; Collier, 2009).

Este estudio supone una contribución al conocimiento sobre gestión de crisis sanitarias en el ámbito turístico y comporta implicaciones para las ONTs y otras entidades gestoras de destinos como las Organizaciones de Marketing de los Destinos. El estudio sobre la comunicación de crisis ante la Covid-19 de las ONTs de España e Italia ha demostrado la importancia de que estas entidades informen correctamente sobre la gestión que realizan durante la crisis sanitaria a través de Twitter, ya que los turistas o usuarios agradecen, les gusta y comparten especialmente estos contenidos y lo demuestran con sus reacciones.

Finalmente, en la línea de investigaciones anteriores el estudio señala la importancia del uso de Twitter para la comunicación de crisis sanitarias (Chew; Eysenbach, 2010), pero también demuestra que en ocasiones se puede desaprovechar su potencial de rapidez (Brummette; Sisco, 2015) si la entidad no comunica sobre el tema al momento o su potencial interactivo para crear diálogo, que es tan importante en crisis sanitarias de este tipo y que generan tanta incertidumbre (Gui et al., 2017).

\section{Limitaciones del estudio}

Las limitaciones del estudio son diversas. En primer lugar, solo se ha analizado un medio social, Twitter. A pesar de ser el medio social más utilizado en situaciones de crisis, sería interesante hacer la comparativa con la comunicación de las ONTs en otros medios sociales, como Facebook o Instagram.

Además, en futuras investigaciones se podría analizar comparativamente las informaciones y contenidos en los medios sociales con las de las noticias en los medios de comunicación tradicionales, para observar las diferencias en tratamiento de los temas, cobertura, etc.

Otra limitación del estudio es no haber podido entrevistar a los gestores de la comunicación de las ONTs de España e Italia, que podrían haber aportado riqueza de informaciones sobre estrategias de comunicación y contenido durante la crisis sanitaria.

\section{Referencias}

Ansa (2020). "Crece turismo, extranjeros superan a locales". Ansa Latina, 10 febrero.

http://www.ansalatina.com/americalatina/noticia/italia/2019/02/10/crece-turismo-extranjeros-superan-a-locales_55 78389e-9310-438c-beb7-0a7263aa02a4.html

Araña, Jorge E.; León, Carmelo J. (2008). "The impact of terrorism on tourism demand”. Annals of tourism research, v. 35, n. 2, pp. 299-315.

https://doi.org/10.1016/j.annals.2007.08.003

Arroyo, Jesús (2020). “Coronavirus: infectados en España y la evolución del brote desde el origen”. Redacción médica, 23 marzo.

https://www.redaccionmedica.com/secciones/sanidad-hoy/coronavirus-infectados-espana-y-evolucion-covid19-desde-origen-4148

Boin, Arjen; Lagadec, Patrick (2000). "Preparing for the future: Critical challenges in crisis management". Journal of contingencies and crisis management, v. 8, n. 4, pp. 185-191.

https://doi.org/10.1111/1468-5973.00138 
Browne, Annie; St-Onge Ahmad, Sacha; Beck, Charles R.; Nguyen-Van-Tam, Jonathan S. (2016). "The roles of transportation and transportation hubs in the propagation of influenza and coronaviruses: a systematic review". Journal of travel medicine, v. 23, n. 1, tav002.

https://doi.org/10.1093/jtm/tav002

Brummette, John; Sisco, Hilary-Fussel (2015). "Using Twitter as a means of coping with emotions and uncontrollable crises". Public relations review, v. 41, n. 1, pp. 89-96.

https://doi.org/10.1016/j.pubrev.2014.10.009

Capriotti, Paul; Zeler, Ileana; Oliveira, Andrea (2019). "Comunicación dialógica 2.0 en Facebook. Análisis de la interacción en las organizaciones de América Latina”. Revista latina de comunicación social, v. 74, pp. 1094-1113.

https://doi.org/10.4185/RLCS-2019-1373

Casero-Ripollés, Andreu (2020). "Impact of Covid-19 on the media system. Communicative and democratic consequences of news consumption during the outbreak". El profesional de la información, v. 29, n. 2, e290223.

https://doi.org/10.3145/epi.2020.mar.23

Cheng, Yang (2020). "The social-mediated crisis communication research: Revisiting dialogue between organizations and publics in crises of China". Public relations review, v. 46, n. 1, pp. 1-11.

https://doi.org/10.1016/j.pubrev.2019.04.003

Chew, Cynthia; Eysenbach, Gunther (2010). "Pandemics in the age of Twitter: content analysis of Tweets during the 2009 H1N1 outbreak". PloS one, v. 5, n. 11, e14118.

https://doi.org/10.1371/journal.pone.0014118

Colley, Kay L.; Collier, Amy (2009). "An overlooked social media tool? Making a case for wikis”. Public relations strategist, v. 19, n. 2, pp. 110-122.

https://www.econbiz.de/Record/an-overlooked-social-media-tool-making-a-case-for-wikis-colley-kay/10008261844

Coombs, W. Timothy (1998). "Analytic framework for crisis situations: Better responses from a better understanding of the situation". Journal of public relations research, v. 10, n. 3, pp. 177-191.

https://doi.org/10.1207/s1532754xjprr1003_02

Coombs, W. Timothy (2007). "Protecting organization reputations during a crisis: The development and application of situational crisis communication theory". Corporate reputation review, v. 1, n. 3, pp. 163-176.

https://doi.org/10.1057/palgrave.crr.1550049

Coombs, W. Timothy (2015). "The value of communication during a crisis: Insights from strategic communication research". Business horizons, v. 58, n. 2, pp. 141-148.

https://doi.org/10.1016/j.bushor.2014.10.003

EFE (2020). “Hace un mes Italia descubría al 'paciente uno' y ahora será dado de alta”. La vanguardia, 21 marzo. https://www.lavanguardia.com/vida/20200321/474287051397/paciente-uno-italia-recuperado-coronavirus.htm/

Europa Press (2019). “España es el segundo país del mundo que más turistas recibe: 82,7 millones". 20 minutos, 27 septiembre.

https://www.20minutos.es/noticia/3779875/0/espana-segundo-pais-mundo-turistas-recibe-millones

Fowler, Brooke M. (2017). "Stealing thunder and filling the silence: Twitter as a primary channel of police crisis communication". Public relations review, v. 43, n. 4, pp. 718-728.

https://doi.org/10.1016/j.pubrev.2017.04.007

Freberg, Karen; Palenchar, Michael J.; Veil, Shari R. (2013). “Managing and sharing H1N1 crisis information using social media bookmarking services". Public relations review, v. 39, n. 3, pp. 178-184.

https://doi.org/10.1016/j.pubrev.2013.02.007

Fuchs, Galia; Reichel, Arie (2011). "An exploratory inquiry into destination risk perceptions and risk reduction strategies of first time vs. repeat visitors to a highly volatile destination". Tourism management, v. 32, n. 2, pp. 266-276.

https://doi.org/10.1016/j.tourman.2010.01.012

Gardner, Lauren M.; Chughtai, Abrar A.; Maclntyre, C. Raina (2016). "Risk of global spread of Middle East respiratory syndrome coronavirus (MERS-CoV) via the air transport network". Journal of travel medicine, v. 23, n. 6 , taw063. https://doi.org/10.1093/jtm/taw063

Granville, Francesca; Mehta, Amisha; Pike, Steven (2016). “Destinations, disasters and public relations: Stakeholder engagement in multi-phase disaster management". Journal of hospitality and tourism management, v. 28, pp. 73-79. https://doi.org/10.1016/j.jhtm.2016.02.001

Gui, Xinning; Kou, Yubo; Pine, Kathleen H.; Chen, Yuanan (2017). “Managing uncertainty: using social media for risk assessment during a public health crisis". In: Conference on human factors in computing systems, pp. 4520-4533. 
Henderson, Joan C.; Ng, Alex (2004). "Responding to crisis: severe acute respiratory syndrome (SARS) and hotels in Singapore". International journal of tourism research, v. 6, n. 6, pp. 411-419.

https://doi.org/10.1002/jtr.505

Huertas, Assumpció; Setó-Pàmies, Dolors; Míguez-González, María-Isabel (2015). "Social media communications strategies of tourist destinations". El profesional de la información, v. 24, n. 1, pp. 15-21.

https://doi.org/10.3145/epi.2015.ene.02

Hung, Kevin K. C.; Mark, Carman K. M.; Yeung, May P. S.; Chan, Emily Y. Y.; Graham, Colin A. (2018). "The role of the hotel industry in the response to emerging epidemics: a case study of SARS in 2003 and H1N1 swine flu in 2009 in Hong Kong". Globalization and health, v. 14, n. 1, article 117.

https://doi.org/10.1186/s12992-018-0438-6

Jha, Ayan; Lin, Leesa; Short, Sarah Massin; Argentini, Giorgia; Gamhewage, Gaya; Savoia, Elena (2018). "Integrating emergency risk communication (ERC) into the public health system response: Systematic review of literature to aid formulation of the 2017 WHO Guideline for ERC policy and practice". PloS one, v. 13, n. 10, e0205555.

https://doi.org/10.1371/journal.pone.0205555

Jin, Yan; Liu, Brooke-Fisher (2010). "The blog-mediated crisis communication model: Recommendations for responding to influential external blogs". Journal of public relations research, v. 22, n. 4, pp. 429-455.

https://doi.org/10.1080/10627261003801420

Joo, Heesoo; Henry, Ronald E.; Lee, Yeon-Kyeng; Berro, Andre D.; Maskery, Brian A. (2019). "The effects of past SARS experience and proximity on declines in numbers of travelers to the Republic of Korea during the 2015 MERS outbreak: A retrospective study". Travel medicine and infectious disease, v. 30, pp. 54-66.

https://doi.org/10.1016/j.tmaid.2019.05.009

Kim, Samuel-Seongseop; Chun, Hejin; Lee, Heesung (2005). "The effects of SARS on the Korean hotel industry and measures to overcome the crisis: A case study of six Korean five-star hotels". Asia Pacific journal of tourism research, v. 10, n. 4, pp. 369-377.

https://doi.org/10.1080/10941660500363694

Lee, Chien-Chian; Chang, Chun-Ping (2008). "Tourism development and economic growth: A closer look at panels". Tourism management, v. 29, n. 1, pp. 180-192.

https://doi.org/10.1016/j.tourman.2007.02.013

Lee, Choong-Ki; Song, Hak-Jun; Bendle, Lawrence J.; Kim, Myung-Ja; Han, Heesup (2012). "The impact of non-pharmaceutical interventions for 2009 H1N1 influenza on travel intentions: A model of goal-directed behavior". Tourism management, v. 33, n. 1, pp. 89-99.

https://doi.org/10.1016/j.tourman.2011.02.006

Liu, Brooke-Fisher; Austin, Lucinda; Jin, Yan (2011). “How publics respond to crisis communication strategies: The interplay of information form and source". Public relations review, v. 37, n. 4, pp. 345-353.

https://doi.org/10.1016/j.pubrev.2011.08.004

Liu, Brooke-Fisher; Kim, Sora (2011). "How organizations framed the 2009 H1N1 pandemic via social and traditional media: Implications for US health communicators". Public relations review, v. 37, n. 3, pp. 233-244.

https://doi.org/10.1016/j.pubrev.2011.03.005

Maciel-Lima, Sandra-Maria; Rasia, José-Miguel; Bagatelli, Rodrigo-Cechelero; Gontarski, Giseli; Colares, Máximo-José D. (2015). "The impact that the influenza A (H1N1) pandemic had on news reporting in the state of Paraná, Brazil". História, ciências, saúde-manguinhos, v. 22, n. 1, pp. 273-291.

https://doi.org/10.1590/S0104-59702015000100016

Marco, Ricard-Morant; López, Arantxa Martín (2010). “El lenguaje afectivo durante la crisis sanitaria de la gripe A. Disparidades". Revista de antropología, v. 65, n. 2, pp. 427-444.

https://doi.org/10.3989/rdtp.2010.14

Marco, Ricard-Morant; López, Arantxa-Martín (2011). "La metáfora bélica durante la crisis sanitaria de la gripe A". Círculo de lingüística aplicada a la comunicación, v. 46, pp. 91-112.

https://doi.org/10.5209/rev_CLAC.2011.v46.3

McKercher, Bob; Chon, Kaye (2004). "The over-reaction to SARS and the collapse of Asian tourism". Annals of tourism research, v. 31, n. 3, pp. 716-719.

https://doi.org/10.1016/j.annals.2003.11.002

Molina, Carlos (2020). “España rozó los 84 millones de turistas en 2019”. Cinco días, 21 enero. https://cincodias.elpais.com/cincodias/2020/01/20/economia/1579518415_556581.html 
Oliveira, Andrea; Huertas, Assumpció (2019). "How do destinations use Twitter to recover their images after a terrorist attack?". Journal of destination marketing and management, v. 12, pp. 46-54.

https://doi.org/10.1016/j.jdmm.2019.03.002

Reisinger, Yvette; Mavondo, Felix (2005). "Travel anxiety and intentions to travel internationally: Implications of travel risk perception". Journal of travel research, v. 43, n. 3, pp. 212-225.

https://doi.org/10.1177/0047287504272017

Richter, Linda K.; Waugh-Jr, William L. (1986). "Terrorism and tourism as logical companions". Tourism management, v. 7, n. 4, pp. 230-238.

https://doi.org/10.1016/0261-5177(86)90033-6

Ritchie, Brent W.; Dorrell, Humphrey; Miller, Daniela; Miller, Graham A. (2004). "Crisis communication and recovery for the tourism industry: Lessons from the 2001 foot and mouth disease outbreak in the United Kingdom". Journal of travel \& tourism marketing, v. 15, n. 2-3, pp. 199-216.

https://doi.org/10.1300/J073v15n02_11

Rittichainuwat, Bongkosh-Ngamsom; Chakraborty, Goutam (2009). "Perceived travel risks regarding terrorism and disease: The case of Thailand". Tourism management, v. 30, n. 3, pp. 410-418.

https://doi.org/10.1016/j.tourman.2008.08.001

Romenti, Stefania; Murtarelli, Grazia; Valentini, Chiara (2014). “Organisations’ conversations in social media: applying dialogue strategies in times of crises". Corporate communications: an international journal, v. 19, n. 1, pp. 10-33.

https://doi.org/10.1108/CCIJ-05-2012-0041

Saffer, Adam J.; Sommerfeldt, Erich J.; Taylor, Maureen (2013). "The effects of organizational Twitter interactivity on organization-public relationships". Public relations review, v. 39, n. 3, pp. 213-215.

https://doi.org/10.1016/j.pubrev.2013.02.005

Saura, Gemma (2020). “¿Por qué Italia y España?”. La vanguardia, 21 abril.

https://www.lavanguardia.com/internacional/20200405/48314311965/por-que-espana-italia.html

Schultz, Friederike; Utz, Sonja; Göritz, Anja (2011). "Is the medium the message? Perceptions of and reactions to crisis communication via Twitter, blogs and traditional media”. Public relations review, v. 37, n. 1, pp. 20-27.

https://doi.org/10.1016/j.pubrev.2010.12.001

Sérvulo-González, Jesús; Salvatierra, Javier (2020). "Habrá que guardar la distancia un tiempo, hasta en la playa". El país, 12 abril.

https://elpais.com/economia/2020-04-11/reyes-maroto-habra-que-guardar-la-distancia-un-tiempo-hasta-en-la-playa.html

Sevillano, Elena (2020). "La OMS declara el brote de coronavirus pandemia global". El país, 11 marzo.

https://elpais.com/sociedad/2020-03-11/la-oms-declara-el-brote-de-coronavirus-pandemia-global.html

Shi, Wenming; Li, Kevin X. (2017). "Impact of unexpected events on inbound tourism demand modeling: evidence of Middle East Respiratory Syndrome outbreak in South Korea". Asia Pacific journal of tourism research, v. 22, n. 3, pp. 344-356.

https://doi.org/10.1080/10941665.2016.1250795

Signorini, Alessio; Segre, Alberto-Maria; Polgreen, Philip M. (2011). "The use of Twitter to track levels of disease activity and public concern in the US during the influenza A H1N1 pandemic". PloS one, v. 6, n. 5, e19467.

https://doi.org/10.1371/journal.pone.0019467

Sweetser, Kaye; Metzgar, Emily (2007). "Communicating during crisis: Use of blogs as a relationship management tool". Public relations review, v. 33, n. 3, pp. 340-342.

https://doi.org/10.1016/j.pubrev.2007.05.016

Thelwall, Mike; Levitt, Jonathan M. (2020). "Retweeting Covid-19 disability issues: Risks, support and outrage". El profesional de la información, v. 29, n. 2, e290216.

https://doi.org/10.3145/epi.2020.mar.16

Tori, Manuel (2020). "Un mes de confinamiento en Italia en cinco hitos”. Público, 21 marzo. https://www.publico.es/internacional/coronavirus-mes-confinamiento-italia-cinco-hitos.html

Torres-Salinas, Daniel (2020). “Ritmo de crecimiento diario de la producción científica sobre Covid-19. Análisis en bases de datos y repositorios en acceso abierto". El profesional de la información, v. 29, n. 2, e290215.

https://doi.org/10.3145/epi.2020.mar.15

Ulmer, Robert; Seeger, Matthew W.; Sellnow, Timothy L. (2007). "Post-crisis communication and renewal: expanding the parameters of post-crisis discourse". Public relations review, v. 33, n. 2, pp. 130-34.

https://doi.org/10.1016/j.pubrev.2006.11.015 
Ulmer, Robert R.; Sellnow, Timothy L.; Seeger, Matthew W. (2015). Effective crisis communication: Moving from crisis to opportunity. London, UK: Sage Publications. ISBN: 9781412980340

Uşaklı, Ahmet; Koç, Burcu; Sönmez, Sevil (2017), “How 'social' are destinations? Examining European DMO social media usage". Journal of destination marketing \& management, v. 6, n. 2, pp. 136-149.

https://doi.org/10.1016/j.jdmm.2017.02.001

Varia, Monali; Wilson, Samantha; Sarwal, Shelly; McGeer, Allison; Gournis, Effie; Galanis, Eleni; Henry, Bonnie (2003). "Investigation of a nosocomial outbreak of severe acute respiratory syndrome (SARS) in Toronto, Canada". Canadian Medical Association journal, v. 169, n. 4, pp. 285-292.

https://www.ncbi.n/m.nih.gov/pmc/articles/PMC180651

Veil, Shari R.; Buehner, Tara; Palenchar, Michael J. (2011). “A work-in-process literature review: Incorporating social media in risk and crisis communication". Journal of contingencies and crisis management, v. 19, n. 2, pp. $110-122$. https://doi.org/10.1111/j.1468-5973.2011.00639.x

Vijaykumar, Santosh; Jin, Yan; Nowak, Glen (2015). "Social media and the virality of risk: The risk amplification through media spread (RAMS) model". Journal of homeland security and emergency management, v. 12, n. 3, pp. 653-677. https://doi.org/10.1515/jhsem-2014-0072

Wang, Bairong; Zhuang, Jun (2017). "Crisis information distribution on Twitter: a content analysis of tweets during hurricane Sandy". Natural hazards, v. 89, n. 1, pp. 161-181.

https://doi.org/10.1007/s11069-017-2960-x

Wang, Yu-Shan (2009). "The impact of crisis events and macroeconomic activity on Taiwan's international inbound tourism demand". Tourism management, v. 30, n. 1, pp. 75-82.

https://doi.org/10.1016/j.tourman.2008.04.010

Wen, Jun; Aston, Joshua; Liu, Xinyi; Ying, Tianyu (2020). "Effects of misleading media coverage on public health crisis: A case of the 2019 novel coronavirus outbreak in China". Anatolia, pp. 1-6.

https://doi.org/10.1080/13032917.2020.1730621

Xifra, Jordi (2020). "Comunicación corporativa, relaciones públicas y gestión del riesgo reputacional en tiempos del Covid-19". El profesional de la información, v. 29, n. 2, e290220.

https://doi.org/10.3145/epi.2020.mar.20

Yang, Yang; Zhang, Hongru; Chen, Xiang (2020). "Coronavirus pandemic and tourism: Dynamic stochastic general equilibrium modeling of infectious disease outbreak". Annals of tourism research, 102913, in press.

https://doi.org/10.1016/j.annals.2020.102913

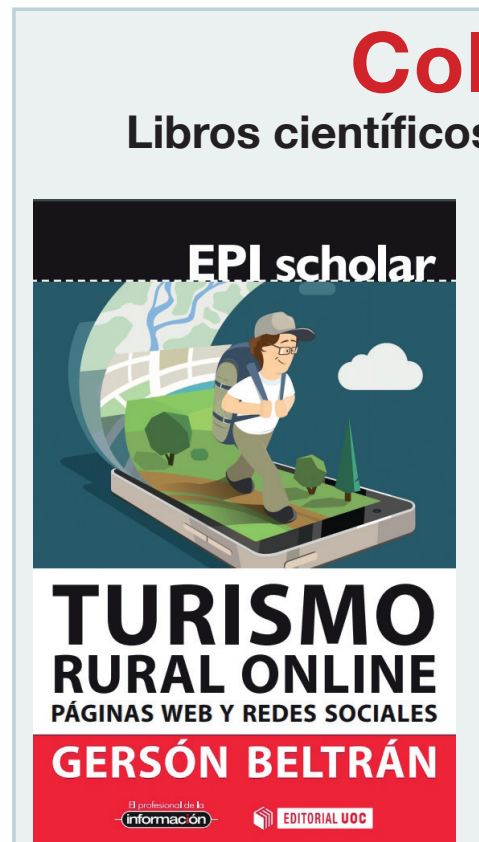

\section{Turismo rural online. Páginas web y redes sociales de Gersón Beltrán}

Los turistas están conectados en todo momento y se mueven en un entorno social, local y móvil: buscan información en internet antes de ir a su destino y realizan las reservas por anticipado, utilizan el móvil durante su experiencia turística para comunicarse y, tanto durante como tras el viaje, comparten sus opiniones en medios sociales.

Las empresas y destinos turísticos deben adaptarse a esta realidad, en la que lo físico es inseparable de lo online, conformando un entorno híbrido. Paralelamente, los espacios rurales sufren numerosos problemas de desarrollo y despoblación, y el turismo rural constituye una actividad que puede ayudar a revertir esta situación. Las nuevas tecnologías se configuran como herramientas que unen a los turistas conectados con estos espacios.

Este libro ofrece una visión general de la situación del turismo rural online, así como las claves para que las empresas y destinos turísticos logren conectar con los turistas y éstos disfruten de las experiencias únicas que les ofrece el turismo rural.

Beltrán, Gersón (2019). Turismo rural online. Páginas web y redes sociales. Barcelona: El profesional de la información, Editorial UOC, colección EPI Scholar n. 11, 170 pp. ISBN: 9788491803881

\section{Información}

http://www.elprofesionaldelainformacion.com/librosEPIScholar.html 